\title{
HARD TO SELECT POINTS IN A PLANE
}

\author{
Thinh D. Nguyen \\ Email: kosmofarmer@yandex.com
}

\begin{abstract}
We show that selecting point in a $2 D$ plane subject to some requirement is a difficult task.
\end{abstract}

\section{Problem Statement}

We are interested in the following optimization problem which is related, in a certain way, to the representation of a set of points using the least number of points among them. More formally, we have a ground set $\mathbf{U}$ of points with coordinates $\left(x_{i}, y_{i}\right)$ for $i=1, \ldots, n$. Given an integer $k \leq n$, the decision proble $\mathrm{m}$ consists in finding a subset of points $\mathbf{I}$ of cardinality $k$ such that every point in $\mathbf{U}$ can be written as the convex combination of at most two points of $\mathbf{I}$.

For example, let $\mathbf{U}=\{(0,0) ;(1,3) ;(2,6) ;(3,0) ;(2,3 / 2) ;(4,3) ;(6,0)\}$. There is a solution with $k=4$ which is $\mathbf{I}=\{(0,0) ;(2,6) ;(4,3) ;(6,0)\}$. Indeed, we have $(1,3)=\frac{(0,0)+(2,6)}{2} ;(2,3 / 2)=\frac{(0,0)+(4,3)}{2}$ and $(3,0)=$ $\underline{(0,0)+(6,0)}$

\section{PROOF OF HARDNESS}

This problem is reducible from VERTEX COVER. Let the input graph to VERTEX COVER be $\mathbf{G}=(\mathbf{V}$, E) with $|\mathbf{V}|=n$. Choose integer $n<t=\mathbf{O}\left(n^{c}\right)$, and create a convex polygon $\mathbf{P}$ on $1+n+2 t$ vertices $\mathbf{C}_{0}$, $\mathbf{C}_{1}, \ldots, \mathbf{C}_{n}, \mathbf{D}_{1}, \ldots, \mathbf{D}_{t}, \mathbf{E}_{1}, \ldots, \mathbf{E}_{t}$. Choose vertices $\mathbf{V}_{1}, \ldots, \mathbf{V}_{n}$ on sides $\mathbf{C}_{0} \mathbf{C}_{1}, \ldots, \mathbf{C}_{n-1} \mathbf{C}_{n}$. Now, for each edge $i j \in \mathbf{E}$ with $i, j \in \mathbf{V}$ and $i<j$, add all points on intersections of $\mathbf{V}_{i} \mathbf{D}_{k}$ and $\mathbf{V}_{j} \mathbf{E}_{l}$ with $1 \leq k, l \leq t$. We choose coordinates in such a way that no point of the plane has more than two diagonals of $\mathbf{P}$ passing through it, and no three intersection points share non-trivial common lines together with vertices $\mathbf{P}$ (other than diagonals of $\mathbf{P}$ when intended).

Obviously, we have to include all vertices of $\mathbf{P}$ into $\mathbf{I}$. Vertices $\mathbf{V}_{i}$ are already covered by sides of $\mathbf{P}$, so we have freedom of including any subset of them. For an edge $i j \in \mathbf{E}$, we have to cover all intersections of $\mathbf{V}_{i} \mathbf{D}_{k}$ and $\mathbf{V}_{j} \mathbf{E}_{l}$ either by including one of $\left\{\mathbf{V}_{i}, \mathbf{V}_{j}\right\}$ into $\mathbf{I}$, or take some of the intersections into $\mathbf{I}$; in the latter case, $\boldsymbol{\Omega}(t)$ intersections need to be included, which effectively prohibits us from doing this. We can now see that the minimal size of $\mathbf{I}$ is exactly $1+n+2 t+\mathbf{V C}(\mathbf{G})$, where $\mathbf{V C}(\mathbf{G})$ is the size of minimal vertex cover of $\mathbf{G}$.

The last remark is that we have to make sure that it is possible to choose all coordinates so that length of coordinates of each point is polynomial of $n$, which is technical.

\section{CONCLUSION}

Garey and Johnson [1] shape their theory based on previous primal works of Cook, Levin and Karp. Johnson [2] moves on with the guide to this theory. As long as we study a mathematical conjecture, we should encourage ourselves to have moderate amount of labor hours on popular mathematics books like these. Then, reading some articles on theory of computing like [3] is a good practice. Having all those done, we could think of the ultimate final for all mathematics sciences.

\section{REFERENCE}


1. Michael R. Garey, David S. Johnson, Computers and Intractability: A Guide to the The ory of NP-Completeness

2. David S. Johnson, The NP-Completeness Column: An Ongoing Guide

3. Phan Dinh Dieu, Le Cong Thanh, Le Tuan Hoa, Average Polynomial Time Complexity of Some NP-Complete Problems, Theor. Comput. Sci. 46(3): 219-237 (1986) 